\title{
A Review on Various Approaches of Data Sensing and Transmission in WSN
}

\author{
Gurleen $^{1}$, Naseeb Singh Dhillon ${ }^{2}$ \\ Research Scholar, Computer Science Department, Adesh Institute of Engineering \& Technology, Faridkot ${ }^{1}$ \\ Assistant Professor, Computer Science Department, Adesh Institute of Engineering \& Technology, Faridkot ${ }^{2}$
}

\begin{abstract}
WSN is the branch of sensing information from the external environment where human interaction is not possible. In WSN various approaches have been used for data sensing and transmission over the networks. In this paper various researches has been reviewed for extraction of valuable information for efficient energy management and data transmission in WSN. In this paper various approaches that are cluster based, relay based and chain based has been illustrated. Relay based WSN use a relay node for receiving information from the sensor node that cause problem in large scale networks. On the basis of analysis of these different approaches dynamic clustering approach provides efficient energy utilization in WSN.
\end{abstract}

Keywords: LEACH, BEC, LEACH-M, C-LEACH, MWSN and Radio Propagation.

\section{INTRODUCTION}

\subsection{Wireless Sensor Network}

A wireless sensor network is a group of specialized transducers with a communications infrastructure for monitoring and recording conditions at diverse locations. Commonly monitored parameters are temperature, humidity, pressure, wind direction and speed, illumination intensity, vibration intensity, sound intensity, power-line voltage, chemical concentrations, pollutant levels and vital body functions [6].The more modern networks are bi-directional, also enabling control of sensor activity. The development of wireless sensor networks was motivated by military applications such as battlefield surveillance; today such networks are used in many industrial and consumer applications, such as industrial process monitoring and control, machine health monitoring [7].

\subsection{Parts of WSN:}

1.2.1 Sensor Node:This is a core component of WSN. This node plays a multiple roles in WSN, such as simple sensing; data storage; routing; and data processing [10].

1.2.2 Clusters: Clusters are the organizational unit for WSNs. The dense nature of these networks requires the need for them to be broken down into clusters to simplify tasks such a communication.

1.2.3 Cluster heads: Cluster heads are the managing the cluster head. They often are needed to managing task in the cluster. These tasks include but are not limited to data-aggregation and organizing the communication schedule of a cluster [11].

1.2.4 Base Station: The base station is at the upper level of the hierarchical WSN. It provides the communication link between the sensor network and the end-user.

1.2.5 End User: The data in a sensor network can be used for a wide-range of applications. Therefore, a particular application may make use of the network data over the internet, using a PDA, or even a desktop computer [15].

\subsection{Clustering}

A free meaning of Clustering could be "the procedure of arranging articles into gatherings whose individuals are comparable somehow". A cluster is along these lines an accumulation of items which are "comparable" in the middle of them and are "divergent" to the articles having a place with different cluster [1].

Clustering is a division of information into gatherings of comparable articles. Speaking to theinformation by less clusters essentially loses certain fine subtle elements, however accomplishesd is entanglement. It displays information by its clusters. Information displaying places clustering in a verifiable point of view established in arithmetic, insights, and numerical investigation.From a machine learning point of view groups compare to concealed examples, thehunt down bunches is unsupervised learning, and the subsequent framework speaks to a information idea [4]. From a handy point of view clustering assumes a remarkable part in information mining applications, for example, logical information investigation, data recovery what's more; content mining, spatial database applications, Web investigation, CRM, showcasing, therapeutic diagnostics, computational science, and numerous others [13]. 


\subsection{Energy Dissipation Model in WSN}

1) Data Acquisition and No Processing (DANP) approach

2) Data Acquisition and Transform Coding (DATC) approach

3) Data Acquisition and Compressive Sensing (DACS) approach

\subsubsection{Compressive Sensing}

Wireless Sensor Networks are comprised of spatially distributed sensor nodes, where each node contains units for sensing, processing, and communicating data. In general, sensor nodes are assumed to have limited processing power and highly constrained energy resources. A typical WSN topology includes a base station - a powerful entity more capable than the ordinary sensor nodes with a significantly higher energy budget. Ordinary sensor nodes transfer processed or raw sensed data to the base station, which performs the final information aggregation and extraction tasks [3].

\subsubsection{Mixed Integrated Programming}

Mixed Integer Programming based analysis of communication networks is extremely useful for uncovering the fundamental performance limits. Choosing an MIP based analysis method has a number of advantages. One of them is the abstraction from a specific protocol which enables us to investigate energy cost in ideal conditions with optimal routing decisions. Secondly, due to global knowledge in the optimization problem solver, the results can be obtained in an efficient and consistent manner.

\subsection{Wireless Sensor Network Applications}

1.5.1 Ecological Data Collection: In natural information accumulation application, are utilized gather different sensor information as a part of a timeframe. If a data to be meaningful so collecting sensor data at regular interval and the nodes would remain at known locations. In the environmental data collection application, a large number of nodes continuously sensing and transmitting data back to a set of base stations that store data using traditional methods [5].

1.5.2. Military Applications: Most of the essential learning of sensor systems is fundamental on the resistance application toward the networks, particularly two vital projects the Distributed Sensor Networks (DSN) and the Sensor Information Technology form the Defence Advanced Research Project Agency (DARPA), sensor systems are connected effectively in the military detecting. Presently remote sensor systems can be an indispensable piece of military charge, control, interchanges, registering, insight, observation, surveillance and focusing on frameworks

1.5.3. Security Monitoring:In it the observing systems are gathered of hubs that are set at settled areas all through a situation that persistently screen one or more sensors to recognize an irregularity. A key distinction between security checking and natural observing is that security systems are not really gathering any information. This significantly affects the ideal system design. Every hub needs to as often as possible check the status of its sensors however it just needs to transmit an information report when there is a security infringement. The quick and solid correspondence of caution messages is the essential framework necessity. These are "report by exemption" systems. It is affirmed that every hub is still present and working[3].

1.5.4. Hub following situations: In which remote sensor system is the following of a labeled article through a territory of space observed by a sensor system. There are numerous condition where one might want to track the area of critical resources or staff. Current stock control frameworks endeavor to track objects by recording the last checkpoint that an article went through. Notwithstanding, with these frameworks it is impractical to decide the present area of an article. For instance, UPS tracks each shipment by examining it with a standardized identification at whatever point it goes through steering focuses. The framework separates when articles don't spill out of checkpoint to checkpoint. In ordinary workplaces it is illogical to anticipate that protests will be consistently gone through checkpoints [8].

1.5.5. Wellbeing Applications Sensor systems: They are likewise generally utilized as a part of social insurance zone. In some present day healing facility sensor systems are built to screen understanding physiological information, to control the medication organization track and screen patients and specialists and inside a doctor's facility. In spring 2004 some clinic in Taiwan even utilize RFID fundamental of above named applications to get the circumstance at direct. Long haul nursing home this application is spotlight on nursing of old individuals.

\section{LITERATURE SURVEY}

Das, $\mathbf{T}$ et al this paper proposing Target tracking is one of the most popular applications of mobile wireless sensor networks (MWSNs), where coverage and data gathering algorithms are foundations to achieve successful target tracking. Since the mobility of sensor nodes is of great importance in this particular class of application, it is crucial to design efficient techniques that can manage the mobility. In this paper, author particularly concentrates on military surveillance area where intruder tracking is one of the critical tasks. Many mobility models proposed so far in the literature are used to simulate and evaluate the performance of the networks. At times group mobility model is preferred over individual mobility model to simulate the nature of the application such as military surveillance, disaster relief, tactical environment etc. Usually, groups are formed at the time of sensor node deployment and become fixed. 
However, this is not appropriate for the application scenario under consideration. This motivates us to design a Cooperative Group Mobility model (CGM) where groups of nodes are formed dynamically.

Zhou Xin-lian et al proposingthis paper presents one inner-cluster scheduling algorithm, avoiding mobile nodes' location affect, satisfying expected coverage scale and high-effect. This excludes the number of smallest inner-cluster active nodes $\mathrm{k}$, which can satisfy expected coverage scale in monitored area, according to coverage analysis theory. In inner-cluster, only select $\mathrm{k}$ nodes with higher energy and nearer close to fixed node (should be avoided inner-cluster nodes leaving), others should be sleeping. Consequently realizes the schedule of higher energy nodes round sleeping. Simulation result display by this schedule, EDG (Efficient Data Gathering) decreases data delay, and largely relieves the burden of cluster-head, and has apparent energy-saving effect, and thinks about node's mobility, can preferably suit to mobile wireless sensor network.

Gagneja, K. K et al proposed Heterogeneous Sensor Networks are more powerful and efficient than homogeneous sensor networks. Homogeneous sensor networks perform poorly because of routine limits and scalability. In this research, author considers to use heterogeneous topology to securely route data in a wireless sensor network. The given area of interest is initially partitioned into Voronoi clusters, where low-end nodes make clusters with high-end nodes. Each cluster has just one high-end node and a number of low-end nodes. Voronoi clusters are driven by the distance between the nodes, but purposed routing method the "Improved Tree Routing" uses hop count to route the data in the network. However, Voronoi clusters leave out some gaps in the topology.

Jambli, M.N. et al this paper proposing saving energy is a very critical issue in wireless sensor networks (WSNs) because sensor nodes have severe resource constraints such as lack of processing power and limited in power supply. Since the communication is the most energy consuming activities in WSNs, the power use for transmission or reception of packet should be managed properly. Transmission power control (TPC) technique is one of the techniques to reduce energy consumption which has been widely studied in mobile ad-hoc networks (MANETs). This technique is implemented by adjusting the transmission power in communication between nodes. However, as mobile wireless sensor networks (WSNs) applications emerge, the unique characteristics of this network such as severe resource constraints and frequent topology change suggest that TPC might be useful to reduce energy consumption in WSN.

\section{APPROACHES USED}

Leach: LEACH is a hierarchical protocol in which most nodes transmit to cluster heads, and the cluster heads aggregate and compresses the data and forward it to the base station (sink). Each node uses a stochastic algorithm at each round to determine whether it will become a cluster head in this round. LEACH assumes that each node has a radio powerful enough to directly reach the base station or the nearest cluster head, but that using this radio at full power all the time would waste energy.Nodes that have been cluster heads cannot become cluster heads again for $\mathrm{P}$ rounds, where $\mathrm{P}$ is the desired percentage of cluster heads. Thereafter, each node has a $1 / \mathrm{P}$ probability of becoming a cluster head again. At the end of each round, each node that is not a cluster head selects the closest cluster head and joins that cluster. The cluster head then creates a schedule for each node in its cluster to transmit its data.

C-Leach:In LEACH-C, clusters forming in the beginning of every period are done, using the centralized algorithm by the base station. The base station uses received information from nodes that includes energy and node status, uses this information during the setup phase for finding pre- determined number of cluster heads and network con- figuration within the clusters. Next classification of nodes in the clusters is done to minimize energy consumption in order to transfer their data to the related cluster head. Results show that LEACH-C overall performance is better than LEACH because of the optimal forming of clusters by the base station. In addition, the number of cluster heads in each period of LEACH-C is equal to the expected optimal value. While in LEACH the number of cluster heads varies in different periods because of lack of global coordination.

LEACH-M (Mobile Low Energy Adaptive Clustering Hierarchy): LEACH-M protocol was proposed for mobility issue in LEACH protocol. This protocol provides mobility to the both non-cluster head nodes and cluster head nodes while the set-up and the steady-state. Nodes are homogeneous and location of each node is calculated by GPS. The nodes with minimum mobility and the lowest attenuation are being selected as cluster head nodes and the role of cluster head nodes is broadcasted to all nodes within its transmission range

HEED: HEED (Hybrid Energy Efficient Distributed) protocol is the clustering protocol. It uses using residual energy as primary parameter and network topology features (e.g. node degree, distances to neighbors) are only used as secondary parameters to break tie between candidate cluster heads, as a metric for cluster selection to achieve load balancing. In this all nodes are assumed to be homogenous i.e. all sensor nodes are equipped with same initial energy. the lifetime of sensor networks is limited there is a need to re-energize the sensor network by adding more nodes. These nodes will be equipped with more energy than the nodes that are already in use, which creates heterogeneity in terms of node energy, leads to the introduction of $\mathrm{H}$.

DEEC: (Distributed Energy Efficient Clustering) In DEEC protocol all nodes use the initial and residual energy level to define the cluster heads. DEEC estimate the ideal value of network lifetime to compute the reference energy that 
each node should expend during each round. In a two-level heterogeneous network, where we have two categories of nodes, $\mathrm{m} . \mathrm{N}$ advanced nodes with initial energy equal to Eo. $(1+\mathrm{a})$ and $(1-\mathrm{m}) . \mathrm{N}$ normal nodes, where the initial energy is equal to Eo.

BEC: the design of Binary Exponential CODE (BEC) back-off MAC protocol is presented and discussed elaborately. The key idea is to move the channel contention among random access stations into a code space of orthogonal codes dynamically to allow parallel transmissions of the competing communications on the same spectrum. Unlike the traditional IEEE 802.11 MAC scheme which transmits sequentially in time domain, our proposed BEC protocol allow multiple parallel transmissions at the same time but with lower data rates. Thiswould address the problems of performance penalty and channel utilization inefficiency incurred by the temporal binary exponential back-off because the channel contention significantly impact the frame transmission because they are serialized in time domain and it is difficult to achieve coordination among random access competing stations to win channel.

\section{CONCLUSION}

Clustering is the most popular topology control method to reduce energy consumption and improve scalability of WSNs. Energy is the main constraint of wireless sensor networks (WSNs) due to irreplaceable and limited power sources of the sensor nodes. in a cluster based WSN, cluster heads (CHs) consume more energy due to extra work load owing to data collection, data aggregation and their communication to the base station. Therefore, efficient cluster formation is very challenging by considering the energy consumption of the CHs. various approaches have been used for formation of efficient clustering in WSN so that energy consumption can be reduced. Maximum amount of energy that consumed in WSN is during data transmission. To overcome this problem an approach must be developed that can sense changes occurred in the current sensed value and previous sensed value, so that redundant information can be blocked.

\section{REFERENCES}

[1] K. Musale, "Analysis of Cluster Based Routing Protocol for Mobile Wireless Sensor Network," Int. J. Adv. Trends Computer Sci. Eng., vol. 2, no. 1, pp. 124-129, 2013.

[2] S. Yadav and S. S. Yadav, "Review for Leach Protocol in WSN," Int. J. Recent Dev. Eng. Technol., vol. 2, no. 6, pp. 69-71, 2014

[3] J. D. Gaurkar and K. Dhote, "Review paper on design of distributed Energy Efficient and Reliable Routing Protocol for Wireless Sensor Networks," Int. J. Res. Emerg. Sci. Technol., vol. 1, no. 4, pp. 41-46, 2014

[4] M. Arshad, M. Alsalem, F. a. Siddqui, N. Kamel, and N. M. Saad, "Efficient cluster head selection scheme in Mobile Data Collector based routing protocol,” 2012 4th Int. Conf. Intell. Adv. Syst., pp. 280-284, Jun. 2012.

[5] P. Madhumathy and D. Sivakumar, "Mobile Sink Based Reliable And Energy Efficient Data Gathering Technique For WSN," J. Theor. Appl. Inf. Technol., vol. 61, no. 1, pp. 1-9, 2014.

[6] X. Chen and P. Yu, "Research on hierarchical mobile wireless sensor network architecture with mobile sensor nodes," in 20103 rd International Conference on Biomedical Engineering and Informatics, 2010, vol. 7, pp. 2863-2867.

[7] N. Marriwala and P. Rathee, "An approach to increase the wireless sensor network lifetime," in 2012 World Congress on Information and Communication Technologies, 2012, pp. 495-499.

[8] V. V. Deshpande and A. R. BhagatPatil, "Energy efficient clustering in wireless sensor network using cluster of cluster heads," in 2013 Tenth International Conference on Wireless and Optical Communications Networks (WOCN), 2013, pp. 1-5.

[9] “The Network Simulator ns-2: Documentation." [Online]. Available: http://www.isi.edu/nsnam/ns/ns-documentation.html. [Accessed: 09-May2015].

[10] Ismat, N "Efficient clustering for Mobile Wireless Sensor Networks", IEEE Conf. on Multi-Topic Conference (INMIC), 2014, pp. 110 - 114.

[11] Hong-Ling Shi "Energy Efficient and Fault Tolerant Multicore Wireless Sensor Network: E²MWSN", IEEE Conf. on Wireless Communications, Networking and Mobile Computing (Wi-COM), 2011, pp. $1-4$.

[12] Das, T "Employing cooperative group mobility model for mobile target tracking in MWSN", IEEE Conf. on Applications and Innovations in Mobile Computing (AIMoC), 2015, pp. $55-61$.

[13] Zhou Xin-lian "The Research of Inner-cluster Nodes Scheduling Algorithm in MWSN", IEEE Conf on Information Technology and Applications (IFITA), 2011, pp. $131-134$.

[14] Gagneja, K.K "Heuristic clustering with secured routing in Heterogeneous Sensor Networks" IEEE Conf. on Security and Privacy of Mobile, Wireless, and Sensor Networks (MWSN), 2013, pp. 9- 16.

[15] Jambli, M.N. "Transmission power control in mobile wireless sensor networks: Simulation-based approach", IEEE Conf. on Wireless Communications and Applications (ICWCA 2012),, 2012, pp. 1 - 6 